\title{
Exterior problem for the spherically symmetric isentropic compressible Navier-Stokes equations with density-dependent viscosity
}

Ruxu Lian ${ }^{1,2^{*}}$ and Jian Liü

\section{${ }^{*}$ Correspondence:}

ruxu.lian.math@outlook.com

${ }^{1}$ College of Mathematics and

Information Science, North China

University of Water Resources and

Electric Power, Zhengzhou, 450011,

P.R. China

${ }^{2}$ Institute of Atmospheric Physics,

Chinese Academy of Sciences,

Beijing, 100029, P.R. China

Full list of author information is

available at the end of the article

\begin{abstract}
In this paper, we study the exterior problem for the spherically symmetric isentropic compressible Navier-Stokes equations with density-dependent viscosity coefficients. Under certain assumptions imposed on the initial data, we show that there exists a unique global strong solution to the exterior problem and obtain the regularity of the strong solution. Some ideas and more delicate estimates are introduced to prove these results.
\end{abstract}

MSC: $35 \mathrm{Q} 35 ; 76 \mathrm{D03}$

Keywords: spherically symmetric Navier-Stokes equations; exterior problem; density-dependent viscosity

\section{Introduction}

In general, the $N$-dimensional isentropic compressible Navier-Stokes equations with density-dependent viscosity coefficients read

$$
\left\{\begin{array}{l}
\rho_{t}+\operatorname{div}(\rho \mathbf{U})=0 \\
(\rho \mathbf{U})_{t}+\operatorname{div}(\rho \mathbf{U} \otimes \mathbf{U})+\nabla P(\rho)-\operatorname{div}(\mu(\rho) D(\mathbf{U}))-\nabla(\lambda(\rho) \operatorname{div} \mathbf{U})=0,
\end{array}\right.
$$

where $t \in(0,+\infty)$ is the time and $\mathbf{x} \in R^{N}, \rho>0$ and $u$ denote the density and velocity, respectively. The pressure function is taken as $P(\rho)=\rho^{\gamma}$ with $\gamma>1$, and

$$
D(\mathbf{U})=\frac{\nabla(\mathbf{U})+{ }^{\mathbb{T}} \nabla(\mathbf{U})}{2}
$$

is the strain tensor and $\mu(\rho), \lambda(\rho)$ are the Lamé viscosity coefficients satisfying

$$
\mu(\rho)>0, \quad \mu(\rho)+N \lambda(\rho) \geq 0 .
$$

There is a huge literature on the studies of the compressible Navier-Stokes equations with density-dependent viscosity coefficients. For example, as $\mu(\rho)=1, \lambda(\rho)=\rho^{\beta}$, and

(c) 2016 Lian and Liu. This article is distributed under the terms of the Creative Commons Attribution 4.0 International License (http://creativecommons.org/licenses/by/4.0/), which permits unrestricted use, distribution, and reproduction in any medium, provided you give appropriate credit to the original author(s) and the source, provide a link to the Creative Commons license, and indicate if changes were made. 
$\beta>3$, Vaigant and Kazhikhov [1] established the existence and uniqueness of global strong solution to the two-dimensional Navier-Stokes system of equations for a barotropic compressible viscous fluid in the square. Ducomet and Nečasová [2] proved the existence and uniqueness of global strong solution to the two-dimensional compressible Navier-StokesFourier system with vorticity-type boundary conditions and density-dependent viscosities in any smooth bounded region of $R^{2}$. The mathematical derivations of the viscous Saint-Venant system were addressed in the simulation of flow surface in shallow region $[3,4]$. The physical model of the viscous Saint-Venant system is the prototype model (corresponding to (1.1) with $P(\rho)=\rho^{2}, \mu(\rho)=\rho$, and $\left.\lambda(\rho)=0\right)$, and Bresch and Desjardins proved the existence of solutions for the $2 \mathrm{D}$ shallow water equations $[5,6]$. The wellposedness of solutions to the free boundary value problem with initial finite mass and the flow density being connected with the infinite vacuum either continuously or via jump discontinuity was investigated by many authors, refer to [7-17] and references therein. Mellet and Vasseur [18] considered barotropic compressible Navier-Stokes equations with density-dependent viscosity coefficients that vanish on the vacuum and proved the stability of weak solutions in periodic domain and whole space. The global existence of strong solutions for one-dimensional compressible Navier-Stokes equations was shown by Mellet and Vasseur [19]. Ducomet et al. [20] investigated the Cauchy problem for the equations of selfgravitating motions of a barotropic gas with density-dependent viscosities, where the pressure $P(\rho)$ is not necessarily a monotone function of the density and proved that the Cauchy problem admits a global weak solution. The Cauchy problem for the equations of spherically symmetric motions in $R^{3}$ of a selfgravitating barotropic gas, with possibly non-monotone pressure law, was considered by Ducomet et al. [21], and they also proved the global existence of weak solution. The qualitative behaviors of global solutions and dynamical asymptotics of vacuum states were also considered, for instance, the finite time vanishing of finite vacuum or asymptotical formation of vacuum in large time, the dynamical behaviors of vacuum boundary, the large time convergence to rarefaction wave with vacuum, and the stability of shock profile with large shock strength, refer to [22-27] and references therein.

In this present paper, we consider the exterior problem for the spherically symmetric isentropic compressible Navier-Stokes equations with density-dependent viscosity coefficients and focus on the global existence, uniqueness and regularity of the strong solution, etc. As $P(\rho)=\rho^{\gamma}(\gamma \geq 2), \mu(\rho)=\rho$, and $\lambda(\rho)=0$, we show that the exterior problem admits a unique global strong solution.

The rest part of the paper is arranged as follows. In Section 2, the main results as regards the global existence of strong solution for the spherically symmetric isentropic compressible Navier-Stokes equations are stated. In Section 3, the a priori estimates for strong solution to the exterior problem are established, and in Section 4 the main results are proved.

\section{Notations and main results}

In this present paper, the viscosity terms are assumed to satisfy $\mu(\rho)=\rho$ and $\lambda(\rho)=0$ in (1.1) and the strain tensor is taken as $D(\mathbf{U})=\nabla \mathbf{U}$. The isentropic compressible NavierStokes equations become

$$
\left\{\begin{array}{l}
\rho_{t}+\operatorname{div}(\rho \mathbf{U})=0, \\
(\rho \mathbf{U})_{t}+\operatorname{div}(\rho \mathbf{U} \otimes \mathbf{U})+\nabla P(\rho)-\operatorname{div}(\rho \nabla \mathbf{U})=0 .
\end{array}\right.
$$


The initial data and boundary conditions of (2.1) are imposed as

$$
\left\{\begin{array}{l}
(\rho, \mathbf{U})(\mathbf{x}, 0)=\left(\rho_{0}, \mathbf{U}_{0}\right)(\mathbf{x}), \quad \mathbf{x} \in \Omega \\
\mathbf{U}=0, \quad \text { on } \partial \Omega, \quad \lim _{|\mathbf{x}| \rightarrow+\infty}(\rho, \mathbf{U})(\mathbf{x}, t)=(\bar{\rho}, 0), \quad t \in[0, T]
\end{array}\right.
$$

where $\Omega:=R^{3} / \Omega_{r_{-}}, \Omega_{r_{-}}$is a ball of radius $r_{-}$centered at the origin in $R^{3}$, and $\bar{\rho}>0$ is a constant.

We will investigate the spherically symmetric solution of the system (2.1) in the spherically symmetric exterior domain $\Omega$ in the present paper, so we denote

$$
|\mathbf{x}|=r, \quad \rho(\mathbf{x}, t)=\rho(r, t), \quad \mathbf{U}(\mathbf{x}, t)=u(r, t) \frac{\mathbf{x}}{r}
$$

which gives the following system of equations for $r>0$ :

$$
\left\{\begin{array}{l}
\rho_{t}+(\rho u)_{r}+\frac{2 \rho u}{r}=0 \\
(\rho u)_{t}+\left(\rho u^{2}+\rho^{\gamma}\right)_{r}+\frac{2 \rho u^{2}}{r}-\left(\rho u_{r}\right)_{r}-\rho\left(\frac{2 u}{r}\right)_{r}=0
\end{array}\right.
$$

with the initial data and boundary conditions

$$
\left\{\begin{array}{l}
(\rho, u)(r, 0)=\left(\rho_{0}, u_{0}\right)(r), \quad r \in\left[r_{-},+\infty\right) \\
u\left(r_{-}, t\right)=0, \quad \lim _{r \rightarrow+\infty}(\rho, u)(r, t)=(\bar{\rho}, 0), \quad t \in[0, T]
\end{array}\right.
$$

and the initial data satisfies for some constant $\underline{\rho}>0$

$$
\left\{\begin{array}{l}
r^{2}\left(\rho_{0}-\bar{\rho}\right) \in L^{1} \cap L^{2}\left(\left[r_{-},+\infty\right)\right), \quad \inf _{r \in\left[r_{-},+\infty\right)} \rho_{0}>\rho_{-}>0 \\
r\left(\rho_{0}^{\frac{1}{2}}\right)_{r} \in L^{2} \cap L^{\infty}\left(\left[r_{-},+\infty\right)\right), \quad r^{2} u_{0} \in H^{1}\left(\left[r_{-},+\infty\right)\right)
\end{array}\right.
$$

Next, we give the definition of a weak solution to the exterior problem (2.1)-(2.2).

Definition 2.1 (weak solution) For any $T>0,(\rho, u)$ is said to be a weak solution of the exterior problem (2.1)-(2.2), if $(\rho, u)$ has the following regularities:

$$
\left\{\begin{array}{l}
\rho \geq 0 \text { a.e., } \quad 0<\rho-\bar{\rho} \in L^{\infty}\left([0, T] ; L^{1}(\Omega) \cap L^{2}(\Omega)\right), \sqrt{\rho} \mathbf{U} \in L^{\infty}\left([0, T] ; L^{2}(\Omega)\right) \\
\nabla\left(\rho^{\frac{1}{2}}\right) \in L^{\infty}\left([0, T] ; L^{2}(\Omega)\right), \quad \rho \nabla \mathbf{U} \in L^{2}\left([0, T] ; L^{2}(\Omega)\right)
\end{array}\right.
$$

and equations (2.1) are satisfied in the sense of a distribution. Namely, for all $\varphi \in C_{0}^{\infty}(\bar{\Omega} \times$ $[0, T])$

$$
\int_{\Omega} \rho_{0} \varphi(\mathbf{x}, 0) d \mathbf{x}+\int_{0}^{T} \int_{\Omega} \rho \varphi_{t} d \mathbf{x} d t+\int_{0}^{T} \int_{\Omega} \rho \mathbf{U} \cdot \nabla \varphi d \mathbf{x} d t=0
$$

and for all $\psi=\left(\psi_{1}, \psi_{2}, \psi_{3}\right) \in C_{0}^{\infty}(\bar{\Omega} \times[0, T])$

$$
\begin{aligned}
& \int_{\Omega} \rho_{0} \mathbf{U}_{0} \cdot \psi(\mathbf{x}, 0) d \mathbf{x}+\int_{0}^{T} \int_{\Omega}\left(\sqrt{\rho}(\sqrt{\rho} \mathbf{U}) \cdot \psi_{t}+\sqrt{\rho} \mathbf{U} \otimes \sqrt{\rho} \mathbf{U}: \nabla \psi\right) d \mathbf{x} d t \\
& \quad+\int_{0}^{T} \int_{\Omega} \rho^{\gamma} \operatorname{div} \psi d \mathbf{x} d t-\langle\rho \nabla \mathbf{U}, \nabla \psi\rangle=0,
\end{aligned}
$$


where the diffusion term makes sense as

$$
\begin{aligned}
\langle\rho \nabla \mathbf{U}, \nabla \psi\rangle= & -\int_{0}^{T} \int_{\Omega} \sqrt{\rho}(\sqrt{\rho} \mathbf{U}) \cdot \Delta \psi d \mathbf{x} d t \\
& -2 \int_{0}^{T} \int_{\Omega}(\sqrt{\rho} \mathbf{U}) \cdot\left(\nabla\left(\rho^{\frac{1}{2}}\right) \cdot \nabla\right) \psi d \mathbf{x} d t .
\end{aligned}
$$

Then we can give the main results as follows.

Theorem 2.1 Let $\gamma \geq 2$. Assume that the initial data satisfies (2.6). Then there exists a unique global strong solution $(\rho, u)$ to the exterior problem (2.4)-(2.5) satisfying for $T>0$

$$
\left\{\begin{array}{l}
0<C(T) \leq \rho \leq C, \quad(\rho, u) \in C\left(\left[r_{-},+\infty\right) \times[0, T]\right), \\
(\rho-\bar{\rho})_{r} \in L^{\infty}\left([0, T] ; L^{2}\left(\left[r_{-},+\infty\right)\right) \cap \cap L^{2}\left([0, T] ; L^{2}\left(\left[r_{-},+\infty\right)\right)\right)\right. \\
u \in L^{\infty}\left([0, T] ; H^{1}\left(\left[r_{-},+\infty\right)\right)\right) \cap L^{2}\left([0, T] ; H^{2}\left(\left[r_{-},+\infty\right)\right)\right), \\
u_{t} \in L^{2}\left([0, T] ; L^{2}\left(\left[r_{-},+\infty\right)\right)\right),
\end{array}\right.
$$

here and below $C(T)>0$ denotes the constant dependent on time and $C>0$ denotes the constant independent of time.

If further $r^{2} u_{0} \in H^{2}\left(\left[r_{-},+\infty\right)\right)$, then $(\rho, u)$ satisfies

$$
\left\{\begin{array}{l}
(\rho, u) \in C\left(\left[r_{-},+\infty\right) \times[0, T]\right), \quad \rho_{\tau} \in L^{\infty}\left(0, T ; L^{2}\left(\left[r_{-},+\infty\right)\right)\right) \\
(\rho-\bar{\rho})_{r} \in L^{\infty}\left([0, T] ; L^{2}\left(\left[r_{-},+\infty\right)\right) \cap \cap L^{2}\left([0, T] ; L^{2}\left(\left[r_{-},+\infty\right)\right)\right)\right. \\
u \in L^{\infty}\left([0, T] ; H^{2}\left(\left[r_{-},+\infty\right)\right) \cap \cap L^{2}\left([0, T] ; H^{3}\left(\left[r_{-},+\infty\right)\right)\right)\right. \\
u_{t} \in L^{\infty}\left([0, T] ; L^{2}\left(\left[r_{-},+\infty\right)\right)\right) \cap L^{2}\left([0, T] ; H^{1}\left(\left[r_{-},+\infty\right)\right)\right)
\end{array}\right.
$$

Remark 2.1 Theorem 2.1 holds for the Saint-Venant model for shallow water, i.e., $P(\rho)=$ $\rho^{2}, \mu(\rho)=\rho, \lambda(\rho)=0$.

Remark 2.2 In this paper, we can obtain several estimates in (3.80) and (3.81) which are not uniformly on time, these estimates can be used to get the compactness results for the exterior problem (2.4)-(2.5), but they not be applied to investigate the large time behaviors of the strong solution.

\section{The a priori estimates}

It is convenient to prove Theorem 2.1 in terms of Lagrange coordinates, and the key step is to establish several useful a priori estimates. Take the Lagrange coordinates to transform

$$
x=\int_{r_{-}}^{r} \rho(r, t) r^{2} d r, \quad \tau=t
$$

which maps $(r, t) \in\left[r_{-},+\infty\right) \times R^{+}$into $(x, \tau) \in[0,+\infty) \times R^{+}$. The relation between Lagrangian coordinates and Eulerian coordinates is satisfied by

$$
\frac{\partial x}{\partial r}=\rho r^{2}, \quad \frac{\partial x}{\partial t}=-\rho u r^{2} .
$$


Under the Lagrangian coordinates transform, the exterior problem (2.4)-(2.5) is reformulated to

$$
\left\{\begin{array}{l}
\rho_{\tau}+\rho^{2}\left(r^{2} u\right)_{x}=0 \\
r^{-2} u_{\tau}+\left(\rho^{\gamma}\right)_{x}=\left(\rho^{2}\left(r^{2} u\right)_{x}\right)_{x}-\frac{2 \rho_{x} u}{r}, \\
(\rho, u)(x, 0)=\left(\rho_{0}, u_{0}\right)(x), \quad x \in[0,+\infty), \\
u(0, \tau)=0, \quad \lim _{x \rightarrow+\infty}(\rho, u)=(\bar{\rho}, 0), \quad \tau \in[0,+\infty),
\end{array}\right.
$$

where the initial data satisfies

$$
\left\{\begin{array}{l}
\rho_{0}-\bar{\rho} \in L^{1} \cap L^{2}([0,+\infty)), \quad \inf _{x \in[0,+\infty)} \rho_{0}>\rho_{-}>0 \\
r^{2} \rho_{0 x} \in L^{2} \cap L^{\infty}([0,+\infty)), \quad \frac{1}{\sqrt{r^{2} \rho_{0}}}\left(r^{2} u_{0}\right) \in L^{2}([0,+\infty)) \\
\sqrt{r^{2} \rho_{0}}\left(r^{2} u_{0}\right)_{x} \in L^{2}([0,+\infty)), \\
\frac{1}{\sqrt{r^{2} \rho_{0}}}\left(r^{2} \rho_{0}\left(r^{2} \rho_{0}\left(r^{2} u_{0}\right)_{x}\right)_{x}\right) \in L^{2}([0,+\infty))
\end{array}\right.
$$

First, we are ready to establish the a priori estimates for the solution $(\rho, u)$ to the exterior problem (3.3). First of all, we can establish the following a priori estimates.

Lemma 3.1 Let $T>0$. Under the conditions in Theorem 2.1, we have for the strong solution $(\rho, u)$ to the exterior problem $(3.3)$

$$
\begin{aligned}
\frac{1}{2} \int_{0}^{+\infty} & u^{2} d x+\int_{0}^{+\infty}\left(\frac{1}{\gamma-1}\left(\rho^{\gamma-1}-\bar{\rho}^{\gamma-1}\right)+\bar{\rho}^{\gamma}\left(\rho^{-1}-\bar{\rho}^{-1}\right)\right) d x \\
& +\int_{0}^{\tau} \int_{0}^{+\infty}\left(\frac{2 u^{2}}{r^{2}}+\rho^{2} u_{x}^{2} r^{4}\right) d x d s \\
= & \frac{1}{2} \int_{0}^{+\infty} u_{0}^{2} d x \\
& +\int_{0}^{+\infty}\left(\frac{1}{\gamma-1}\left(\rho_{0}^{\gamma-1}-\bar{\rho}^{\gamma-1}\right)+\bar{\rho}^{\gamma}\left(\rho_{0}^{-1}-\bar{\rho}^{-1}\right)\right) d x, \quad \tau \in[0, T] .
\end{aligned}
$$

Proof Multiplying (3.3) $)_{2}$ by $r^{2} u$ and integrating the result with respect to $x$ over $[0,+\infty)$, making use of $(3.3)_{1}$, we have

$$
\begin{aligned}
\frac{1}{2} & \frac{d}{d \tau} \int_{0}^{+\infty} u^{2} d x+\int_{0}^{+\infty} \rho^{2}\left(r^{2} u\right)_{x}^{2} d x-2 \int_{0}^{+\infty} \rho\left(r u^{2}\right)_{x} d x \\
& =\int_{0}^{+\infty}\left(\rho^{\gamma}-\bar{\rho}^{\gamma}\right)\left(r^{2} u\right)_{x} d x \\
& =\int_{0}^{+\infty}\left(\rho^{\gamma-2}-\bar{\rho}^{\gamma} \rho^{-2}\right)\left(-\rho_{\tau}\right) d x \\
& =-\frac{d}{d \tau} \int_{0}^{+\infty}\left(\frac{1}{\gamma-1}\left(\rho^{\gamma-1}-\bar{\rho}^{\gamma-1}\right)+\bar{\rho}^{\gamma}\left(\rho^{-1}-\bar{\rho}^{-1}\right)\right) d x,
\end{aligned}
$$

integrating (3.6) with respect to $\tau$, we obtain (3.5). 
Lemma 3.2 Let $T>0$. Under the conditions in Theorem 2.1, we have for the strong solution $(\rho, u)$ to the exterior problem (3.3)

$$
\begin{aligned}
\frac{1}{2} \int_{0}^{+\infty}\left(u+r^{2} \rho_{x}\right)^{2} d x+\int_{0}^{+\infty}\left(\frac{1}{\gamma-1}\left(\rho^{\gamma-1}-\bar{\rho}^{\gamma-1}\right)+\bar{\rho}^{\gamma}\left(\rho^{-1}-\bar{\rho}^{-1}\right)\right) d x \\
\quad+\gamma \int_{0}^{\tau} \int_{0}^{+\infty} \rho^{\gamma-1} \rho_{x}^{2} r^{4} d x d s \\
=\frac{1}{2} \int_{0}^{+\infty}\left(u_{0}+r^{2} \rho_{0 x}\right)^{2} d x \\
\quad+\int_{0}^{+\infty}\left(\frac{1}{\gamma-1}\left(\rho_{0}^{\gamma-1}-\bar{\rho}^{\gamma-1}\right)+\bar{\rho}^{\gamma}\left(\rho_{0}^{-1}-\bar{\rho}^{-1}\right)\right) d x, \quad \tau \in[0, T] .
\end{aligned}
$$

Proof Differentiating (3.3) ${ }_{1}$ with respect to $x$, we have

$$
\rho_{x \tau}+\left(\rho^{2}\left(r^{2} u\right)_{x}\right)_{x}=0 .
$$

Summing (3.8) and (3.3) 2 , we have

$$
\left(r^{-2} u+\rho_{x}\right)_{\tau}+\left(\rho^{\gamma}\right)_{x}=\left(r^{-2}\right)_{\tau} u-\frac{2 \rho_{x} u}{r} .
$$

Note that

$$
r^{3}(x, \tau)=r_{-}^{3}+3 \int_{0}^{x} \frac{1}{\rho(z, \tau)} d z,
$$

and so

$$
\frac{\partial r}{\partial \tau}=\frac{1}{r^{2}} \int_{0}^{x}\left(\frac{1}{\rho}\right)_{\tau}(z, \tau) d z=\frac{1}{r^{2}} \int_{0}^{x}\left(r^{2} u\right)_{z}(z, \tau) d z=u(x, \tau),
$$

which together with (3.9) yields

$$
\left(r^{-2} u+\rho_{x}\right)_{\tau}+\left(\rho^{\gamma}\right)_{x}=-2 r^{-3} u^{2}-\frac{2 \rho_{x} u}{r} .
$$

Multiplying (3.12) by $\left(u+r^{2} \rho_{x}\right) r^{2}$, and integrating the result with respect to $x$ and $\tau$, we have (3.7).

Lemma 3.3 Let $T>0$. Under the conditions in Theorem 2.1, we have for the strong solution $(\rho, u)$ to the exterior problem (3.3)

$$
\rho(x, \tau) \leq C, \quad(x, \tau) \in[0,+\infty) \times[0, T]
$$

where $C$ is a positive constant independent of time.

Proof Let

$$
\varphi(\rho):=\frac{1}{\gamma-1}\left(\rho^{\gamma-1}-\bar{\rho}^{\gamma-1}\right)+\bar{\rho}^{\gamma}\left(\rho^{-1}-\bar{\rho}^{-1}\right)
$$


and

$$
\psi(\rho):=\int_{\bar{\rho}}^{\rho} \varphi(\zeta)^{\frac{1}{2}} d \zeta
$$

It follows from (3.5) and (3.15) that

$$
\begin{aligned}
|\psi(\rho)| & =\left|\int_{x}^{+\infty} \partial_{x} \psi(\rho) d x\right|=\left|\int_{x}^{+\infty} \psi^{\prime}(\rho) \rho_{x}(x, \tau) d x\right| \\
& \leq C \int_{0}^{+\infty} \varphi(\rho) d x+C \int_{0}^{+\infty} r^{4} \rho_{x}^{2} d x \leq C .
\end{aligned}
$$

As $\rho \rightarrow+\infty$, we have for some $\theta \in(0,1)$, if $1<\gamma \leq 3$,

$$
\begin{aligned}
& \lim _{\rho \rightarrow+\infty} \psi(\rho) \\
& \quad=\lim _{\rho \rightarrow+\infty} \int_{\bar{\rho}}^{\rho}\left((\gamma-2)(\theta \bar{\rho}+(1-\theta) \eta)^{\gamma-3}+2 \bar{\rho}^{\gamma}(\theta \bar{\rho}+(1-\theta) \eta)^{-3}\right)^{\frac{1}{2}}(\eta-\bar{\rho}) d \eta \\
& \quad \geq \lim _{\rho \rightarrow+\infty}\left((\gamma-2)(\theta \bar{\rho}+(1-\theta) \rho)^{\gamma-3}+2 \bar{\rho}^{\gamma}(\theta \bar{\rho}+(1-\theta) \rho)^{-3}\right)^{\frac{1}{2}} \int_{\bar{\rho}}^{\rho}(\eta-\bar{\rho}) d \eta \\
& \quad=\lim _{\rho \rightarrow+\infty}\left((\gamma-2)(\theta \bar{\rho}+(1-\theta) \rho)^{\gamma-3}+2 \bar{\rho}^{\gamma}(\theta \bar{\rho}+(1-\theta) \rho)^{-3}\right)^{\frac{1}{2}} \cdot \frac{1}{2}(\rho-\bar{\rho})^{2} \\
& \quad \rightarrow+\infty
\end{aligned}
$$

and if $\gamma>3$, we have

$$
\begin{aligned}
& \lim _{\rho \rightarrow+\infty} \psi(\rho) \\
& \quad=\lim _{\rho \rightarrow+\infty} \int_{\bar{\rho}}^{\rho}\left((\gamma-2)(\theta \bar{\rho}+(1-\theta) \eta)^{\gamma-3}+2 \bar{\rho}^{\gamma}(\theta \bar{\rho}+(1-\theta) \eta)^{-3}\right)^{\frac{1}{2}}(\eta-\bar{\rho}) d \eta \\
& \geq \lim _{\rho \rightarrow+\infty}\left((\gamma-2) \bar{\rho}^{\gamma-3}\right)^{\frac{1}{2}} \int_{\bar{\rho}}^{\rho}(\eta-\bar{\rho}) d \eta \\
& \quad=\lim _{\rho \rightarrow+\infty}\left((\gamma-2) \bar{\rho}^{\gamma-3}\right)^{\frac{1}{2}} \cdot \frac{1}{2}(\rho-\bar{\rho})^{2} \\
& \rightarrow+\infty
\end{aligned}
$$

which with (3.16) yields

$$
\rho(x, \tau) \leq C, \quad(x, \tau) \in[0,+\infty) \times[0, T] .
$$

Next, the Lagrangian structure of the particle transport for this exterior problem (3.3) will be shown as follows. Without loss of generality, we define two particle paths $r_{1}(t), r_{2}(t)$ in Eulerian coordinates as

$$
\begin{cases}\frac{d}{d t} r_{1}(t)=u\left(r_{1}(t), t\right), & r_{1}(0)=r_{10}, \\ \frac{d}{d t} r_{2}(t)=u\left(r_{2}(t), t\right), & r_{2}(0)=r_{20}\end{cases}
$$


where $r_{10}$ and $r_{20}$ satisfy

$$
r_{-} \leq r_{10}<r_{20}<+\infty
$$

Since we have the conservation of total mass,

$$
\begin{aligned}
& \int_{r_{-}}^{r_{1}(t)} \rho(r, t) r^{2} d r=\int_{r_{-}}^{r_{10}} \rho_{0}(r) r^{2} d r:=a, \\
& \int_{r_{-}}^{r_{2}(t)} \rho(r, t) r^{2} d r=\int_{r_{-}}^{r_{20}} \rho_{0}(r) r^{2} d r:=b,
\end{aligned}
$$

and the two paths $r_{1}(t), r_{2}(t)$ are transformed into $x=a, x=b$, furthermore, the domain $\left[r_{1}(t), r_{2}(t)\right]$ is transformed into $[a, b]$, where $0 \leq a<b<+\infty$.

Lemma 3.4 Let $T>0$. Under the conditions in Theorem 2.1, we have for the strong solution $(\rho, u)$ to the exterior problem $(3.3)$

$$
\begin{aligned}
& r_{-}+C x^{\frac{\gamma}{3(\gamma-1)}} \leq r(x, \tau) \leq+\infty, \quad(x, \tau) \in[a, b] \times[0, T] \\
& r(b, \tau)-r(a, \tau) \geq C(b-a)^{\frac{\gamma}{3(\gamma-1)}}, \quad 0 \leq a<b<+\infty
\end{aligned}
$$

where $C$ is a positive constant independent of time.

Proof By the Lagrangian coordinates transform (3.1), for any $x \in[a, b]$ and $r_{1}(t) \leq r(x, \tau) \leq$ $r_{2}(t)$, where $r_{-} \leq r_{10} \leq r(x, 0) \leq r_{20}<+\infty$, we can find that

$$
\begin{aligned}
x & =\int_{r_{-}}^{r(x, \tau)} \rho(r, t) r^{2} d r \\
& \leq C\left(\int_{r_{-}}^{r(x, \tau)} \rho^{\gamma}(r, t) r^{2} d r\right)^{\frac{1}{\gamma}}\left(\int_{r_{-}}^{r(x, \tau)} r^{2} d r\right)^{\frac{\gamma-1}{\gamma}} \\
& \leq C\left(\|\rho\|_{L^{\infty}}^{\gamma-1} \int_{r_{-}}^{r(x, \tau)} \rho(r, t) r^{2} d r\right)^{\frac{1}{\gamma}}\left(\int_{r_{-}}^{r(x, \tau)} r^{2} d r\right)^{\frac{\gamma-1}{\gamma}} \\
& \leq C\left(\int_{r_{-}}^{r(x, 0)} \rho_{0}(r) r^{2} d r\right)^{\frac{1}{\gamma}}\left(\int_{r_{-}}^{r(x, \tau)} r^{2} d r\right)^{\frac{\gamma-1}{\gamma}} \\
& \leq C\left(r(x, \tau)-r_{-}\right)^{\frac{3(\gamma-1)}{\gamma}},
\end{aligned}
$$

which implies for $(x, \tau) \in[0,+\infty) \times[0, T]$ that

$$
r(x, \tau) \geq C x^{\frac{\gamma}{3(\gamma-1)}}+r_{-} .
$$

For any $0 \leq a<b<+\infty$, we have

$$
\begin{aligned}
b-a & =\int_{r(a, \tau)}^{r(b, \tau)} \rho(r, t) r^{2} d r \\
& \leq C\left(\int_{r(a, \tau)}^{r(b, \tau)} \rho^{\gamma}(r, t) r^{2} d r\right)^{\frac{1}{\gamma}}\left(\int_{r(a, \tau)}^{r(b, \tau)} r^{2} d r\right)^{\frac{\gamma-1}{\gamma}}
\end{aligned}
$$




$$
\begin{aligned}
& \leq C\left(\|\rho\|_{L^{\infty}}^{\gamma-1} \int_{r(a, \tau)}^{r(b, \tau)} \rho(r, t) r^{2} d r\right)^{\frac{1}{\gamma}}\left(\int_{r(a, \tau)}^{r(b, \tau)} r^{2} d r\right)^{\frac{\gamma-1}{\gamma}} \\
& \leq C\left(\int_{r_{10}}^{r_{20}} \rho_{0}(r) r^{2} d r\right)^{\frac{1}{\gamma}}\left(\int_{r(a, \tau)}^{r(b, \tau)} r^{2} d r\right)^{\frac{\gamma-1}{\gamma}} \\
& \leq C(r(b, \tau)-r(a, \tau))^{\frac{3(\gamma-1)}{\gamma}}
\end{aligned}
$$

which together with

$$
r(b, \tau)-r(a, \tau)=\int_{a}^{b} \frac{1}{\rho(r, t) r^{2}} d r \geq 0
$$

implies

$$
r(b, \tau)-r(a, \tau) \geq C(b-a)^{\frac{\gamma}{3(\gamma-1)}} .
$$

Lemma 3.5 Let $T>0$. Under the conditions in Theorem 2.1, we have for the strong solution $(\rho, u)$ to the exterior problem $(3.3)$

$$
\begin{aligned}
& \int_{0}^{\tau}\left\|\rho^{2(\gamma-1)} u^{2}\right\|_{L^{\infty}([a, b])} d s \leq C(T), \\
& \int_{0}^{\tau}\left(\rho^{\gamma}\right)_{x}^{2} r^{4} d s \leq C(T), \quad(x, \tau) \in[a, b] \times[0, T]
\end{aligned}
$$

where $C(T)$ is a positive constant dependent on time $T$.

Proof From (3.3) we have

$$
\rho_{x} r^{2}=\rho_{0 x} r^{2}+u_{0}-u-\int_{0}^{\tau}\left(\rho^{\gamma}\right)_{x} r^{2} d s .
$$

From (3.33)

$$
\begin{aligned}
\int_{0}^{\tau}\left(\rho^{\gamma}\right)_{x}^{2} r^{4} d s= & \gamma^{2} \int_{0}^{\tau} \rho^{2(\gamma-1)}\left(\rho_{x} r^{2}\right)^{2} d s \\
= & \gamma^{2} \int_{0}^{\tau} \rho^{2(\gamma-1)}\left(\rho_{0 x} r^{2}-u+u_{0}-\int_{0}^{s}\left(\rho^{\gamma}\right)_{x} r^{2} d l\right)^{2} d s \\
\leq & C(T)\left(\int_{0}^{\tau} \rho^{2(\gamma-1)}\left(\rho_{0 x}^{2} r^{4}+u^{2}+u_{0}^{2}\right) d s+\int_{0}^{\tau} \int_{0}^{s}\left(\rho^{\gamma}\right)_{x}^{2} r^{4} d l d s\right) \\
\leq & C(T) \int_{0}^{\tau} \rho^{4(\gamma-1)} d s+C(T) \int_{0}^{\tau} \rho_{0 x}^{4} r^{8} d s+C(T) \int_{0}^{\tau} u_{0}^{2} d s \\
& +C(T) \int_{0}^{\tau}\left\|\rho^{2(\gamma-1)} u^{2}\right\|_{L^{\infty}([a, b])} d s+C(T) \int_{0}^{\tau} \int_{0}^{s}\left(\rho^{\gamma}\right)_{x}^{2} r^{4} d l d s \\
\leq & C(T)+C(T) \int_{0}^{\tau}\left\|\rho^{2(\gamma-1)} u^{2}\right\|_{L^{\infty}([a, b])} d s \\
& +C(T) \int_{0}^{\tau} \int_{0}^{s}\left(\rho^{\gamma}\right)_{x}^{2} r^{4} d l d s .
\end{aligned}
$$


By means of $\gamma \geq 2$, we have

$$
\begin{aligned}
\left\|\rho^{\gamma-1} u\right\|_{L^{\infty}([a, b])} & \leq C \int_{a}^{b}\left|\rho^{\gamma-1} u\right| d x+C \int_{a}^{b}\left|\rho^{\gamma-2} \rho_{x} u\right| d x+C \int_{a}^{b}\left|\rho^{\gamma-1} u_{x}\right| d x \\
& \leq C+C \int_{a}^{b} u^{2} d x+C \int_{a}^{b} \rho_{x}^{2} r^{4} d x+C\left(\int_{a}^{b} \rho^{2} u_{x}^{2} r^{4} d x\right)^{\frac{1}{2}} \\
& \leq C+C\left(\int_{a}^{b} \rho^{2} u_{x}^{2} r^{4} d x\right)^{\frac{1}{2}},
\end{aligned}
$$

which with Gronwall's inequality yields the lemma.

Lemma 3.6 Let $T>0$. Under the conditions in Theorem 2.1, for a small constant $\delta_{1} \in$ $\left(0, \frac{b-a}{2}\right)$

$$
\rho(x, \tau) \geq C\left(\delta_{1}, T\right), \quad(x, \tau) \in\left[a+\delta_{1}, b-\delta_{1}\right] \times[0, T]
$$

where $C\left(\delta_{1}, T\right)$ is a positive constant dependent on $\delta_{1}$ and time T. Furthermore, for a small constant $\delta_{2} \in\left(0, \frac{b}{2}\right)$

$$
\rho(x, \tau) \geq C\left(\delta_{2}, T\right), \quad(x, \tau) \in\left[0, b-\delta_{2}\right] \times[0, T]
$$

where $C\left(\delta_{2}, T\right)$ is a positive constant dependent on $\delta_{2}$ and time $T$.

Proof For a small positive constant $\eta \in\left(0, \frac{b-a}{8}\right)$, we can find two points $x_{1} \in[a+\eta, a+2 \eta]$, $x_{2} \in[a+3 \eta, a+4 \eta]$, and define, for $x_{i}, i=1,2$,

$$
r\left(x_{i}\right):=r_{-}+\int_{0}^{x_{i}} \frac{1}{\rho_{0}(r) r^{2}} d r
$$

meanwhile we have

$$
0<r_{-}<r\left(x_{1}\right)<r\left(x_{2}\right)<+\infty
$$

Define two particle paths $r_{x_{i}}(t)$ as

$$
\begin{aligned}
& \frac{d}{d t} r_{x_{i}}(t)=u\left(r_{x_{i}}(t), t\right), \quad r_{x_{i}}(0)=r\left(x_{i}\right), \quad i=1,2, \\
& r_{x_{i}}(t)=r\left(x_{i}\right)+\int_{0}^{t} u\left(r_{x_{i}}(s), s\right) d s, \quad i=1,2
\end{aligned}
$$

and we have from the conservation of total mass

$$
\int_{r_{x_{1}}(t)}^{r_{x_{2}}(t)} \rho(r, t) r^{2} d r=\int_{r\left(x_{1}\right)}^{r\left(x_{2}\right)} \rho_{0}(r) r^{2} d r=x_{2}-x_{1}>0, \quad t \geq 0
$$

Then we can find a curve in Eulerian coordinates

$$
r=r_{*}(t) \in\left[r_{x_{1}}(t), r_{x_{2}}(t)\right]
$$


defined by

$$
\frac{d}{d t} r_{*}(t)=u\left(r_{*}(t), t\right), \quad r_{*}(0)=r_{*} \in\left[r\left(x_{1}\right), r\left(x_{2}\right)\right],
$$

such that we have

$$
\begin{aligned}
\rho\left(r_{*}(t), t\right) r_{*}^{2}(t) & =\frac{1}{r_{x_{2}}(t)-r_{x_{1}}(t)} \int_{r_{x_{1}}(t)}^{r_{x_{2}}(t)} \rho(r, t) r^{2} d r \\
& =\frac{x_{2}-x_{1}}{r_{x_{2}}(t)-r_{x_{1}}(t)}, \quad t \geq 0 .
\end{aligned}
$$

Furthermore, in Lagrangian coordinates, there exists $x_{*} \in\left[x_{1}, x_{2}\right]$ :

$$
x_{*}:=\int_{r_{-}}^{r_{*}(t)} \rho(r, t) r^{2} d r=\int_{r_{-}}^{r_{*}} \rho_{0}(r) r^{2} d r
$$

such that

$$
\rho\left(x_{*}, \tau\right) r_{*}^{2}\left(x_{*}, \tau\right)=\frac{x_{2}-x_{1}}{r_{x_{2}}(\tau)-r_{x_{1}}(\tau)}, \quad \tau \geq 0 .
$$

In the same way, as $\eta$ is small enough, we can find another two points $x_{3} \in[b-4 \eta, b-3 \eta]$, $x_{4} \in[b-2 \eta, b-\eta]$ and define, for $x_{i}, i=3,4$,

$$
r\left(x_{i}\right):=r_{-}+\int_{0}^{x_{i}} \frac{1}{\rho_{0}(r) r^{2}} d r
$$

which implies

$$
0<r_{-}<r\left(x_{3}\right)<r\left(x_{4}\right)<+\infty
$$

Define two particle paths

$$
\begin{aligned}
& \frac{d}{d t} r_{x_{i}}(t)=u\left(r_{x_{i}}(t), t\right), \quad r_{x_{i}}(0)=r\left(x_{i}\right), \quad i=3,4, \\
& r_{x_{i}}(t)=r\left(x_{i}\right)+\int_{0}^{t} u\left(r_{x_{i}}(s), s\right) d s, \quad i=3,4,
\end{aligned}
$$

and we have from the conservation of total mass

$$
\int_{r_{x_{3}}(t)}^{r_{x_{4}}(t)} \rho(r, t) r^{2} d r=\int_{r\left(x_{3}\right)}^{r\left(x_{4}\right)} \rho_{0}(r) r^{2} d r=x_{4}-x_{3}>0, \quad t \geq 0 .
$$

As by the argument above, there exists a curve in Eulerian coordinates

$$
r=r^{*}(t) \in\left[r_{x_{3}}(t), r_{x_{4}}(t)\right],
$$

defined by

$$
\frac{d}{d t} r^{*}(t)=u\left(r^{*}(t), t\right), \quad r^{*}(0)=r^{*} \in\left[r\left(x_{1}\right), r\left(x_{2}\right)\right],
$$


meanwhile, in Lagrangian coordinates there exists $x^{*} \in\left[x_{3}, x_{4}\right]$ :

$$
x^{*}:=\int_{r_{-}}^{r^{*}(t)} \rho(r, t) r^{2} d r=\int_{r_{-}}^{r^{*}} \rho_{0}(r) r^{2} d r
$$

such that

$$
\rho\left(x^{*}, \tau\right) r^{* 2}\left(x^{*}, \tau\right)=\frac{x_{4}-x_{3}}{r_{x_{4}}(\tau)-r_{x_{3}}(\tau)} .
$$

Set $v(x, \tau)=\frac{1}{\rho(x, \tau) r^{2}(x, \tau)}$ and define

$$
V(T):=\max _{\left[x_{*}, x^{*}\right] \times[0, T]} v(x, \tau) .
$$

From $(3.3)_{1}$, we have

$$
v_{\tau}=\frac{\left(r^{2} u\right)_{x}}{r^{2}}-\frac{2 v u}{r} .
$$

For any $\beta>1$, multiplying (3.58) by $\beta v^{\beta-1}$, and integrating the equation over $\left[x_{*}, x^{*}\right] \times$ $[0, \tau]$, we have

$$
\int_{0}^{\tau} \int_{x_{*}}^{x^{*}} \frac{d}{d s} v^{\beta} d x d s=\beta \int_{0}^{\tau} \int_{x_{*}}^{x^{*}} v^{\beta-1} \frac{\left(r^{2} u\right)_{x}}{r^{2}} d x d s-2 \beta \int_{0}^{\tau} \int_{x_{*}}^{x^{*}} \frac{v^{\beta} u}{r} d x d s,
$$

which yields

$$
\begin{aligned}
\int_{x_{*}}^{x^{*}} v^{\beta} d x & =\int_{x_{*}}^{x^{*}} v_{0}^{\beta} d x+\beta \int_{0}^{\tau} \int_{x_{*}}^{x^{*}} v^{\beta-1} \frac{\left(r^{2} u\right)_{x}}{r^{2}} d x d s-2 \beta \int_{0}^{\tau} \int_{x_{*}}^{x^{*}} \frac{v^{\beta} u}{r} d x d s \\
& :=\int_{x_{*}}^{x^{*}} v_{0}^{\beta} d x+I_{1}+I_{2},
\end{aligned}
$$

where

$$
\begin{aligned}
I_{1}= & \left.\beta \int_{0}^{\tau} v^{\beta-1} u(x, s)\right|_{x=x_{*}} ^{x=x^{*}} d s-\beta(\beta-1) \int_{0}^{\tau} \int_{x_{*}}^{x^{*}} v^{\beta-2} v_{x} r^{2} u d x d s \\
& +2 \beta \int_{0}^{\tau} \int_{x_{*}}^{x^{*}} \frac{v^{\beta} u}{r} d x d s \\
= & \left.\beta \int_{0}^{\tau} v^{\beta-1} u(x, s)\right|_{x=x_{*}} ^{x=x^{*}} d s+\beta(\beta-1) \int_{0}^{\tau} \int_{x_{*}}^{x^{*}} v^{\beta} u \rho_{x} r^{2} d x d s \\
& +2 \beta(\beta-1) \int_{0}^{\tau} \int_{x_{*}}^{x^{*}} \frac{v^{\beta} u}{r} d x d s+2 \beta \int_{0}^{\tau} \int_{x_{*}}^{x^{*}} \frac{v^{\beta} u}{r} d x d s \\
= & \left.\beta \int_{0}^{\tau} v^{\beta-1} u(x, s)\right|_{x=x_{*}} ^{x=x_{*}^{*}} d s \\
& +\beta(\beta-1) \int_{0}^{\tau} \int_{x_{*}}^{x^{*}} v^{\beta} u\left(\rho_{0 x} r^{2}-u+u_{0}-\int_{0}^{s}\left(\rho^{\gamma}\right)_{x} r^{2} d l\right) d x d s \\
& +2 \beta(\beta-1) \int_{0}^{\tau} \int_{x_{*}}^{x^{*}} \frac{v^{\beta} u}{r} d x d s+2 \beta \int_{0}^{\tau} \int_{x_{*}}^{x^{*}} \frac{v^{\beta} u}{r} d x d s
\end{aligned}
$$


and

$$
I_{2}=-2 \beta \int_{0}^{\tau} \int_{x_{*}}^{x^{*}} \frac{v^{\beta} u}{r} d x d s
$$

Namely

$$
\begin{aligned}
& \int_{x_{*}}^{x^{*}} v^{\beta} d x+\beta(\beta-1) \int_{0}^{\tau} \int_{x_{*}}^{x^{*}} v^{\beta} u^{2} d x d s \\
& =\int_{x_{*}}^{x^{*}} v_{0}^{\beta} d x+\beta(\beta-1) \int_{0}^{\tau} \int_{x_{*}}^{x^{*}} v^{\beta} u \rho_{0 x} r^{2} d x d s+\beta(\beta-1) \int_{0}^{\tau} \int_{x_{*}}^{x^{*}} v^{\beta} u u_{0} d x d s \\
& \quad-\beta(\beta-1) \int_{0}^{\tau} \int_{x_{*}}^{x^{*}} v^{\beta} u\left(\int_{0}^{s}\left(\rho^{\gamma}\right)_{x} r^{2} d l\right) d x d s \\
& \quad+\left.\beta \int_{0}^{\tau} v^{\beta-1} u(x, s)\right|_{x=x_{*}} ^{x=x^{*}} d s+2 \beta(\beta-1) \int_{0}^{\tau} \int_{x_{*}}^{x^{*}} \frac{v^{\beta} u}{r} d x d s \\
& :=J_{1}+J_{2}+J_{3}+J_{4}+J_{5}+J_{6} .
\end{aligned}
$$

A complicated computation gives

$$
\begin{aligned}
& J_{1 \leq C,} \\
& \begin{aligned}
J_{2}+J_{3} \leq & \frac{\beta(\beta-1)}{6} \int_{0}^{\tau} \int_{x_{*}}^{x^{*}} v^{\beta} u^{2} d x d s+C \int_{0}^{\tau} \int_{x_{*}}^{x^{*}} v^{\beta} \rho_{0 x}^{2} r^{4} d x d s \\
& +C \int_{0}^{\tau} \int_{x_{*}}^{x^{*}} v^{\beta} u_{0}^{2} d x d s \\
\leq & \frac{\beta(\beta-1)}{6} \int_{0}^{\tau} \int_{x_{*}}^{x^{*}} v^{\beta} u^{2} d x d s+C \int_{0}^{\tau} \int_{x_{*}}^{x^{*}} v^{\beta} d x d s
\end{aligned}
\end{aligned}
$$

and

$$
\begin{aligned}
J_{4} & \leq \frac{\beta(\beta-1)}{6} \int_{0}^{\tau} \int_{x_{*}}^{x^{*}} v^{\beta} u^{2} d x d s+C \int_{0}^{\tau} \int_{x_{*}}^{x^{*}} v^{\beta}\left(\int_{0}^{s}\left(\rho^{\gamma}\right)_{x} r^{4} d l\right)^{2} d x d s \\
& \leq \frac{\beta(\beta-1)}{6} \int_{0}^{\tau} \int_{x_{*}}^{x^{*}} v^{\beta} u^{2} d x d s+C(T) \int_{0}^{\tau} \int_{x_{*}}^{x^{*}} v^{\beta} d x d s .
\end{aligned}
$$

Next, we will give the estimate of the term $J_{5}$. We have

$$
\begin{aligned}
v\left(x_{*}, \tau\right) & =\frac{1}{\rho\left(x_{*}, \tau\right) r^{2}\left(x_{*}, \tau\right)} \\
& =\frac{r_{x_{2}}(\tau)-r_{x_{1}}(\tau)}{x_{2}-x_{1}} \\
& \leq \eta^{-1} r_{x_{2}}(\tau) \\
& \leq \eta^{-1} r_{x_{2}}(0)+\eta^{-1} \int_{0}^{\tau}\left|u\left(r_{x_{2}}(s), s\right)\right| d s \\
& \leq \eta^{-1} r_{x_{2}}(0)+\eta^{-1} C(T)\left(\int_{0}^{\tau}\|u\|_{L^{\infty}([a, b])}^{2} d s\right)^{\frac{1}{2}} .
\end{aligned}
$$


In the same way, we can obtain

$$
\begin{aligned}
v\left(x^{*}, \tau\right) & =\frac{1}{\rho\left(x^{*}, \tau\right) r^{2}\left(x^{*}, \tau\right)}=\frac{r_{x_{4}}(\tau)-r_{x_{3}}(\tau)}{x_{4}-x_{3}} \\
& \leq \eta^{-1} r_{x_{4}}(\tau) \leq \eta^{-1} r_{x_{4}}(0)+\eta^{-1} C(T)\left(\int_{0}^{\tau}\|u\|_{L^{\infty}([a, b])}^{2} d s\right)^{\frac{1}{2}},
\end{aligned}
$$

meanwhile, we know that

$$
\begin{aligned}
\int_{0}^{\tau}\|u\|_{L^{\infty}([a, b])}^{2} d s & \leq C \int_{0}^{\tau} \int_{a}^{b} u^{2} d x d s+C \int_{0}^{\tau} \int_{a}^{b}\left|u u_{x}\right| d x d s \\
& \leq C(T)+C\left(\int_{0}^{\tau} \int_{a}^{b} \rho^{2} u_{x}^{2} r^{4} d x d s\right)^{\frac{1}{2}}\left(\int_{0}^{\tau} \int_{a}^{b} v^{2} u^{2} d x d s\right)^{\frac{1}{2}} \\
& \leq C(T)+C(T) V(T) .
\end{aligned}
$$

From (3.67)-(3.69), we have

$$
\begin{aligned}
J_{5} & \leq \beta \int_{0}^{\tau}\left|v^{\beta-1} u\left(x_{*}, s\right)\right| d s+\beta \int_{0}^{\tau}\left|v^{\beta-1} u\left(x^{*}, s\right)\right| d s \\
& \leq C(T) \eta^{-(\beta-1)}\left(1+\int_{0}^{\tau}\|u\|_{L^{\infty}([a, b])}^{2} d s\right)^{\frac{\beta-1}{2}} \int_{0}^{\tau}\|u\|_{L^{\infty}([a, b])} d s \\
& \leq C(T) \eta^{-(\beta-1)}\left(1+\int_{0}^{\tau}\|u\|_{L^{\infty}([a, b])}^{2} d s\right)^{\frac{\beta}{2}} \\
& \leq C(T) \eta^{-(\beta-1)}\left(1+V(T)^{\frac{\beta}{2}}\right)
\end{aligned}
$$

and

$$
J_{6} \leq \frac{\beta(\beta-1)}{6} \int_{0}^{\tau} \int_{x_{*}}^{x^{*}} \nu^{\beta} u^{2} d x d s+C \int_{0}^{\tau} \int_{x_{*}}^{x^{*}} \nu^{\beta} d x d s .
$$

Finally, we obtain

$$
\int_{x_{*}}^{x^{*}} v^{\beta} d x \leq C(T) \int_{0}^{\tau} \int_{x_{*}}^{x^{*}} v^{\beta} d x d s+C(T) \eta^{-(\beta-1)} V(T)^{\frac{\beta}{2}}+C(T) \eta^{-(\beta-1)}+C .
$$

Applying Gronwall's inequality, for $\tau \in[0, T]$

$$
\int_{x_{*}}^{x^{*}} v^{\beta} d x \leq C(\eta, T)\left(1+V(T)^{\frac{\beta}{2}}\right)
$$

Let $\delta_{1}:=4 \eta$, for $x \in\left[a+\delta_{1}, b-\delta_{1}\right]$

$$
\begin{aligned}
v(x, \tau)^{\beta} & \leq \frac{1}{x^{*}-x_{*}} \int_{x_{*}}^{x^{*}} v^{\beta} d x+\int_{x_{*}}^{x^{*}}\left|\left(v^{\beta}\right)_{x}\right| d x \\
& \leq C(\eta, T)+C(\eta, T) V(T)^{\frac{\beta}{2}}+C \int_{x_{*}}^{x^{*}} v^{\beta+1}\left|\rho_{x}\right| r^{2} d x
\end{aligned}
$$




$$
\begin{aligned}
& \leq C(\eta, T)+C(\eta, T) V(T)^{\frac{\beta+1}{2}}+C\left(\int_{x_{*}}^{x^{*}} v^{2(\beta+1)} d x\right)^{\frac{1}{2}}\left(\int_{x_{*}}^{x^{*}} \rho_{x}^{2} r^{4} d x\right)^{\frac{1}{2}} \\
& \leq C(\eta, T)+C(\eta, T)+V(T)^{\frac{\beta+1}{2}} .
\end{aligned}
$$

By Young's inequality, we have

$$
V(T) \leq C\left(\delta_{1}, T\right)
$$

which yields

$$
\rho(x, \tau) \geq C\left(\delta_{1}, T\right), \quad(x, \tau) \in\left[a+\delta_{1}, b-\delta_{1}\right] \times[0, T] .
$$

Furthermore, repeating the above arguments with few modifications on the domain $[0, b]$, we can prove (3.37). The details are omitted here. The proof is completed.

Lemma 3.7 Let $T>0$. Under the conditions in Theorem 2.1, we have for the strong solution $(\rho, u)$ to the exterior problem (3.3)

$$
\rho(x, \tau) \geq C(T), \quad(x, \tau) \in[0,+\infty) \times[0, T]
$$

where $C(T)$ is a positive constant dependent on time $T$.

Proof By means of $\rho \rightarrow \bar{\rho}$ as $x \rightarrow+\infty$, we know that $\exists M>0$ such that

$$
\rho(x, \tau) \geq C_{1}, \quad x \in[M,+\infty)
$$

where $C_{1}$ is a positive constant independent of $T$. We apply Lemma 3.6 on the domain $\left[0, M+\delta_{2}\right] \times[0, T]$ with $\delta_{2} \in\left(0, \frac{M}{2}\right)$ a constant small enough, and we can obtain

$$
\rho(x, \tau) \geq C_{2}(T), \quad x \in[0, M]
$$

where $C_{2}(T)$ is a positive constant dependent on $T$. The proof is completed.

Lemma 3.8 Let $T>0$. Under the conditions in Theorem 2.1, we have for the strong solution $(\rho, u)$ to the exterior problem (3.3)

$$
\begin{aligned}
& \int_{0}^{+\infty}\left(r^{2} u\right)_{x}^{2} d x+\int_{0}^{\tau} \int_{0}^{+\infty}\left(r^{2} u\right)_{s}^{2} r^{-4} d x d s+\int_{0}^{\tau} \int_{0}^{+\infty}\left(r^{2} u\right)_{x x}^{2} d x d s \leq C(T), \\
& \int_{0}^{+\infty}\left(r^{2} u\right)_{\tau}^{2} r^{-4} d x+\int_{0}^{\tau} \int_{0}^{+\infty} \rho^{2}\left(r^{2} u\right)_{x s}^{2} d x d s \leq C(T), \quad \tau \in[0, T]
\end{aligned}
$$

where $C(T)>0$ denotes a constant dependent on time.

Proof Multiplying (3.3) $)_{2}$ by $\rho^{-2}\left(r^{2} u\right)_{\tau}$ and integrating the result with respect to $x$ over $[0,+\infty)$, making use of $(3.4)$, we obtain 
Sian and Diu Boundary Value Problems (2016) 2016:49

Page 16 of 20

$$
\begin{aligned}
\frac{d}{d \tau} \int_{0}^{+\infty}\left(\frac{1}{2}\left(r^{2} u\right)_{x}^{2}-\rho^{\gamma-2}\left(r^{2} u\right)_{x}\right) d x+\int_{0}^{+\infty} \rho^{-2}\left(r^{2} u\right)_{\tau}^{2} r^{-4} d x \\
=(\gamma-2) \int_{0}^{+\infty} \rho^{\gamma-1}\left(r^{2} u\right)_{x}^{2} d x-2 \int_{0}^{+\infty} \rho^{\gamma-3} \rho_{x}\left(r^{2} u\right)_{\tau} d x \\
\quad+2 \int_{0}^{+\infty} \rho^{-1} \rho_{x}\left(r^{2} u\right)_{x}\left(r^{2} u\right)_{\tau} d x \\
\quad+2 \int_{0}^{+\infty} \rho^{-2} u^{2}\left(r^{2} u\right)_{\tau} r^{-3} d x-2 \int_{0}^{+\infty} \rho^{-2} \rho_{x} u\left(r^{2} u\right)_{\tau} r^{-1} d x
\end{aligned}
$$

which implies

$$
\begin{aligned}
& \int_{0}^{+\infty}\left(r^{2} u\right)_{x}^{2} d x+\int_{0}^{\tau} \int_{0}^{+\infty}\left(r^{2} u\right)_{s}^{2} r^{-4} d x d s \\
& \leq C+C \int_{0}^{+\infty}\left(\rho^{\gamma-2}-\bar{\rho}^{\gamma-2}\right)^{2} d x+C \int_{0}^{\tau} \int_{0}^{+\infty}\left(\frac{u^{2}}{r^{2}}+u_{x}^{2} r^{4}\right) d x d s \\
& \quad+C(T) \int_{0}^{\tau} \int_{0}^{+\infty} \rho_{x}^{2} r^{4} d x d s+C \int_{0}^{\tau} \int_{0}^{+\infty} \rho_{x}^{2}\left(r^{2} u\right)_{x}^{2} r^{4} d x d s \\
& \quad+C(T) \int_{0}^{\tau} \int_{0}^{+\infty} u^{4} r^{-2} d x d s+C \int_{0}^{\tau} \int_{0}^{+\infty} \rho_{x}^{2} u^{2} r^{2} d x d s \\
& \leq C(T)+C(T) \int_{0}^{\tau} \int_{0}^{+\infty} \rho_{x}^{2}\left(r^{2} u\right)_{x}^{2} r^{4} d x d s+C(T) \sup _{\tau \in[0, T]}\|u\|_{L^{\infty}}^{2} .
\end{aligned}
$$

From $(3.3)_{2},(3.5),(3.7),(3.13)$, and (3.77), we can deduce that for some small $\epsilon \in(0,1)$

$$
\begin{aligned}
& \int_{0}^{\tau} \int_{0}^{+\infty} \rho_{x}^{2}\left(r^{2} u\right)_{x}^{2} r^{4} d x d s \\
& \leq \epsilon \int_{0}^{\tau} \int_{0}^{+\infty} \rho_{x}^{2}\left(r^{2} u\right)_{x}^{2} r^{4} d x d s+\epsilon \int_{0}^{\tau} \int_{0}^{+\infty}\left(r^{2} u\right)_{s}^{2} r^{-4} d x d s \\
& \quad+\epsilon \int_{0}^{\tau} \int_{0}^{+\infty} \rho_{x}^{2} d x d s+C(T) \int_{0}^{\tau} \int_{0}^{+\infty}\left(\frac{u^{2}}{r^{2}}+\rho^{2} u_{x}^{2} r^{4}\right) d x d s, \\
& \sup _{\tau \in[0, T]}\|u\|_{L^{\infty}}^{2} \leq \epsilon \sup _{\tau \in[0, T]} \int_{0}^{+\infty}\left(r^{2} u\right)_{x}^{2} d x+C(T) \sup _{\tau \in[0, T]}^{+\infty} \int_{0}^{2} d x,
\end{aligned}
$$

using (3.83)-(3.85), we can obtain

$$
\begin{aligned}
& \int_{0}^{+\infty}\left(r^{2} u\right)_{x}^{2} d x+\int_{0}^{\tau} \int_{0}^{+\infty}\left(r^{2} u\right)_{s}^{2} r^{-4} d x d s \\
& \quad \leq C(T)+C(T) \int_{0}^{\tau} \int_{0}^{+\infty}\left(\frac{u^{2}}{r^{2}}+\rho^{2} u_{x}^{2} r^{4}\right) d x d s \\
& \quad \leq C(T) .
\end{aligned}
$$

Differentiating (3.3) $)_{2}$ with respect to $\tau$, multiplying the result by $\left(r^{2} u\right)_{\tau}$ and integrating the result with respect to $x$ over $[0,+\infty)$, we have

$$
\begin{aligned}
& \frac{1}{2} \frac{d}{d \tau} \int_{0}^{+\infty}\left(r^{2} u\right)_{\tau}^{2} r^{-4} d x+\int_{0}^{+\infty} \rho^{2}\left(r^{2} u\right)_{x \tau}^{2} d x \\
& \quad=2 \int_{0}^{+\infty} u u_{\tau}\left(r^{2} u\right)_{\tau} r^{-3} d x-\frac{1}{2} \int_{0}^{+\infty}\left(r^{-4}\right)_{\tau}\left(r^{2} u\right)_{\tau}^{2} d x+2 \int_{0}^{+\infty} u\left(r^{-1} u\right)_{\tau}\left(r^{2} u\right)_{\tau} r^{-2} d x
\end{aligned}
$$




$$
\begin{aligned}
& +\int_{0}^{+\infty}\left(\rho^{\gamma}\right)_{\tau}\left(r^{2} u\right)_{x \tau} d x-\int_{0}^{+\infty}\left(\rho^{2}\right)_{\tau}\left(r^{2} u\right)_{x}\left(r^{2} u\right)_{x \tau} d x \\
& -\int_{0}^{+\infty}\left(\frac{2 \rho_{x} u}{r}\right)_{\tau}\left(r^{2} u\right)_{\tau} d x+2 \int_{0}^{+\infty}\left(r^{-2}\right)_{\tau} r^{-1} u^{2}\left(r^{2} u\right)_{\tau} d x
\end{aligned}
$$

A complicated computation gives

$$
\begin{aligned}
& \frac{d}{d \tau} \int_{0}^{+\infty}\left(r^{2} u\right)_{\tau}^{2} r^{-4} d x+\int_{0}^{+\infty} \rho^{2}\left(r^{2} u\right)_{x \tau}^{2} d x \\
& \leq C\left(1+\int_{0}^{+\infty}\left(\frac{u^{2}}{r^{2}}+\rho^{2} u_{x}^{2} r^{4}\right) d x\right) \int_{0}^{+\infty}\left(r^{2} u\right)_{\tau}^{2} r^{-4} d x \\
& \quad+C \sup _{\tau \in[0, T]}\left\|\left(r^{2} u\right)_{x}^{2}\right\|_{L^{\infty}} \int_{0}^{+\infty}\left(r^{2} u\right)_{x}^{2} d x+C \int_{0}^{+\infty}\left(\frac{u^{2}}{r^{2}}+u_{x}^{2} r^{4}\right) d x
\end{aligned}
$$

and by means of Gronwall's inequality, (3.3) 2 , (3.5), (3.7), (3.13), (3.77), and (3.86), we have

$$
\begin{aligned}
& \sup _{\tau \in[0, T]} \int_{0}^{+\infty}\left(r^{2} u\right)_{\tau}^{2} r^{-4} d x+\int_{0}^{\tau} \int_{0}^{+\infty} \rho^{1+\alpha}\left(r^{2} u\right)_{x s}^{2} d x d s \\
& \quad \leq C(T)+C(T) \sup _{\tau \in[0, T]}\left\|\left(r^{2} u\right)_{x}^{2}\right\|_{L^{\infty}} \\
& \quad \leq C(T)+C(T) \sup _{\tau \in[0, T]}\left(\int_{0}^{+\infty}\left(r^{2} u\right)_{x}^{2} d x\right)^{\frac{1}{2}}\left(\int_{0}^{+\infty}\left(r^{2} u\right)_{x x}^{2} d x\right)^{\frac{1}{2}} \\
& \quad \leq C(T)+C(T)+\frac{1}{2} \sup _{\tau \in[0, T]} \int_{0}^{+\infty}\left(r^{2} u\right)_{\tau}^{2} r^{-4} d x,
\end{aligned}
$$

we can complete the proof of Lemma 3.8 .

Remark 3.9 By Lemmas 3.1-3.8, the following inequality holds:

$$
\begin{aligned}
& \int_{0}^{+\infty}(\rho-\bar{\rho})^{2} d x+\int_{0}^{+\infty} \rho_{x}^{2} d x+\int_{0}^{+\infty} u^{2} d x+\int_{0}^{+\infty} u_{x}^{2} d x+\int_{0}^{+\infty} u_{\tau}^{2} d x \\
& \quad+\int_{0}^{\tau} \int_{0}^{+\infty} \rho_{x}^{2} d x d s+\int_{0}^{\tau} \int_{0}^{+\infty} u_{x}^{2} d x d s+\int_{0}^{\tau} \int_{0}^{+\infty} u_{s}^{2} d x d s \\
& \quad+\int_{0}^{\tau} \int_{0}^{+\infty} u_{x x}^{2} d x d s+\int_{0}^{\tau} \int_{0}^{+\infty} u_{x s}^{2} d x d s \leq C
\end{aligned}
$$

\section{Proof of the main results}

Proof Let $\left(\rho_{0}, u_{0}\right)$ be the initial data as described in the theorem, and define $\rho_{0}^{\delta}:=j_{\delta} * \rho_{0}$, $u_{0}^{\delta}:=j_{\delta} * u_{0}$, where $j_{\delta}=\delta^{-1} j(x / \delta)$ is the standard mollifier. Then, for any $0<\beta<1$, $\rho_{0}^{\delta} \in C^{1+\beta}([0,+\infty))$ and $u_{0}^{\delta} \in C^{2+\beta}([0,+\infty))$, which implies that as $\delta \rightarrow 0, \rho_{0}^{\delta} \rightarrow \rho_{0}$ in $W^{1,2}([0,+\infty)), u_{0}^{\delta} \rightarrow u_{0}$ in $L^{2}([0,+\infty))$.

Next, we consider the Cauchy problem (3.3) with the initial data $\left(\rho_{0}, u_{0}\right)$ replaced by $\left(\rho_{0}^{\delta}, u_{0}^{\delta}\right)$, using the energy estimates and the contraction mapping theorem, we can obtain the existence of a unique local solution $\left(\rho^{\delta}, u^{\delta}\right)$ with $\rho^{\delta}, \rho_{x}^{\delta}, \rho_{\tau}^{\delta}, \rho_{\tau x}^{\delta}, u^{\delta}, u_{x}^{\delta}, u_{\tau}^{\delta}$, $u_{x x}^{\delta} \in C^{\beta, \beta / 2}\left([0,+\infty) \times\left[0, T^{*}\right]\right)$ for some $T^{*}>0$. Furthermore, from Lemmas 3.1-3.8, we see that $\rho^{\delta}$ is pointwise bounded from below and above, $u^{\delta}, \rho_{x}^{\delta} \in L^{\infty}\left([0, T] ; L^{2}([0,+\infty))\right)$, 
$u_{x}^{\delta} \in L^{2}\left([0, T] ; L^{2}([0,+\infty))\right), \rho^{\delta}, \rho_{x}^{\delta}, \rho_{\tau}^{\delta}, \rho_{\tau x}^{\delta}, u^{\delta}, u_{x}^{\delta}, u_{\tau}^{\delta}, u_{x x}^{\delta} \in C^{\beta, \beta / 2}([0,+\infty) \times[0, T])$ for any $T>0$. Therefore, we can continue the local solution globally in time and deduce that there exists a unique global solution $\left(\rho^{\delta}, u^{\delta}\right)$ of the Cauchy problem (3.3) with $\left(\rho_{0}, u_{0}\right)$ replaced by $\left(\rho_{0}^{\delta}, u_{0}^{\delta}\right)$, which is carried out as in [9].

Thus, extracting a subsequence of $\left(\rho^{\delta}, u^{\delta}\right)$, still denoted by $\left(\rho^{\delta}, u^{\delta}\right)$, such that as $\delta \rightarrow 0$, we have

$$
\begin{aligned}
& u^{\delta} \rightarrow u \text { weak } * \text { in } L^{\infty}\left([0, T] ; L^{2}([0,+\infty))\right), \\
& \rho^{\delta} \rightarrow \rho \text { weak } * \text { in } L^{\infty}\left([0, T] ; L^{2}([0,+\infty))\right), \\
& \left(\rho_{\tau}^{\delta}, u_{x}^{\delta}\right) \rightarrow\left(\rho_{\tau}, u_{x}\right) \text { weakly in } L^{2}\left([0, T] ; L^{2}([0,+\infty))\right) .
\end{aligned}
$$

Moreover, from (3.5), (3.7), (3.13), and (3.77), the global existence of weak solutions of the Cauchy problem (3.3) can be directly proved. As a matter of fact, because of (3.80) and (3.81), $(\rho, u)$ is also a global strong solution.

Next, we will prove the uniqueness of global strong solution as follows: let $\left(\rho_{1}(x, t)\right.$, $\left.u_{1}(x, t)\right)$ and $\left(\rho_{2}(x, t), u_{2}(x, t)\right)$ be two global strong solutions of the exterior problem (3.3) on the time interval $[0, T]$. For convenience, we set

$$
\varrho_{1}(x, \tau)=\frac{1}{\rho_{1}(x, \tau)}, \quad \varrho_{2}(x, \tau)=\frac{1}{\rho_{2}(x, \tau)},
$$

and we have from (3.3)

$$
\varrho_{1 \tau}(x, \tau)=\left(r^{2} u_{1}\right)_{x}, \quad \varrho_{2 \tau}(x, \tau)=\left(r^{2} u_{2}\right)_{x}
$$

Then, from $(3.3)_{2}$, we obtain

$$
r^{-2}\left(u_{1}-u_{2}\right)_{\tau}+\left(\rho_{1}^{\gamma}-\rho_{2}^{\gamma}\right)_{x}=\left(\rho_{1}^{2}\left(r^{2} u_{1}\right)_{x}-\rho_{2}^{2}\left(r^{2} u_{2}\right)_{x}\right)_{x}-\left(\frac{2 \rho_{1 x} u_{1}}{r}-\frac{2 \rho_{2 x} u_{2}}{r}\right) .
$$

Multiplying the above equation by $\left(u_{1}-u_{2}\right) r^{2}$ and integrating the result with respect to $x$ over $[0,+\infty)$, we have

$$
\begin{aligned}
\frac{1}{2} \frac{d}{d \tau} & \int_{0}^{+\infty}\left(u_{1}-u_{2}\right)^{2} d x \\
= & \int_{0}^{+\infty}\left(\varrho_{1}-\varrho_{2}\right)_{\tau}\left(\varrho_{1}^{-\gamma}-\varrho_{2}^{-\gamma}\right) d x-\int_{0}^{+\infty} \varrho_{1}^{-2}\left(\left(r^{2} u_{1}\right)_{x}-\left(r^{2} u_{2}\right)_{x}\right)^{2} d x \\
& -\int_{0}^{+\infty}\left(\varrho_{1}^{-2}-\varrho_{2}^{-2}\right)\left(r^{2} u_{2}\right)_{x}\left(\left(r^{2} u_{1}\right)_{x}-\left(r^{2} u_{2}\right)_{x}\right) d x \\
& +4 \int_{0}^{+\infty} \varrho_{1}^{-1}\left(\left(r^{2} u_{1}\right)_{x}-\left(r^{2} u_{2}\right)_{x}\right)\left(u_{1}-u_{2}\right) r^{-1} d x \\
& +2 \int_{0}^{+\infty}\left(\varrho_{1}^{-1}-\varrho_{2}^{-1}\right)\left(r^{2} u_{2}\right)_{x}\left(u_{1}-u_{2}\right) r^{-1} d x-6 \int_{0}^{+\infty}\left(u_{1}-u_{2}\right)^{2} r^{-2} d x \\
& +2 \int_{0}^{+\infty}\left(\varrho_{1}^{-1}-\varrho_{2}^{-1}\right) u_{2}\left(\left(r^{2} u_{1}\right)_{x}-\left(r^{2} u_{2}\right)_{x}\right) r^{-1} d x \\
\leq & -C_{0} \int_{0}^{+\infty}\left(\left(r^{2} u_{1}\right)_{x}-\left(r^{2} u_{2}\right)_{x}\right)^{2} d x-\frac{d}{d \tau} \int_{0}^{+\infty} a(x, \tau)\left(\varrho_{1}-\varrho_{2}\right)^{2} d x
\end{aligned}
$$




$$
\begin{aligned}
& +\int_{0}^{+\infty} a_{\tau}(x, \tau)\left(\varrho_{1}-\varrho_{2}\right)^{2} d x+C \int_{0}^{+\infty}\left(1+\left|\left(r^{2} u_{2}\right)_{x}\right|\right)^{2}\left(\varrho_{1}-\varrho_{2}\right)^{2} d x \\
& +C \int_{0}^{+\infty}\left(u_{1}-u_{2}\right)^{2} d x
\end{aligned}
$$

where $C_{0}$ and $C$ are positive constants independent of $T$ and $a(x, \tau)$ is defined as follows:

$$
a(x, \tau):=\frac{\gamma}{2} \int_{0}^{1}\left(\varrho_{2}+\theta\left(\varrho_{1}-\varrho_{2}\right)\right)^{-(\gamma+1)} d \theta
$$

which has a positive lower bound on $[0,+\infty) \times[0, T]$. Furthermore, we have

$$
\left|a_{\tau}(x, \tau)\right| \leq C\left(\left|\varrho_{2 \tau}\right|+\left|\varrho_{1 \tau}-\varrho_{2 \tau}\right|\right)
$$

and

$$
\begin{aligned}
& \int_{0}^{+\infty} a_{\tau}(x, \tau)\left(\varrho_{1}-\varrho_{2}\right)^{2} d x \\
& \quad \leq \frac{C_{0}}{2} \int_{0}^{+\infty}\left(\varrho_{1 \tau}-\varrho_{2 \tau}\right)^{2} d x+C \int_{0}^{+\infty}\left(1+\left|\varrho_{2 \tau}\right|\right)\left(\varrho_{1}-\varrho_{2}\right)^{2} d x
\end{aligned}
$$

where $C$ is a positive constant independent of $T$. Then, applying (4.10) and integrating (4.7) over $[0, \tau]$, we obtain

$$
\begin{aligned}
& \frac{1}{2} \int_{0}^{+\infty}\left(u_{1}-u_{2}\right)^{2} d x+\int_{0}^{+\infty} a(x, \tau)\left(\varrho_{1}-\varrho_{2}\right)^{2} d x \\
& \quad+\frac{C_{0}}{2} \int_{0}^{\tau} \int_{0}^{+\infty}\left(\left(r^{2} u_{1}\right)_{x}-\left(r^{2} u_{2}\right)_{x}\right)^{2} d x d s \\
& \leq C \int_{0}^{\tau} \int_{0}^{+\infty}\left(1+\left|\left(r^{2} u_{2}\right)_{x}\right|\right)^{2}\left(\varrho_{1}-\varrho_{2}\right)^{2} d x d s+C \int_{0}^{\tau} \int_{0}^{+\infty}\left(u_{1}-u_{2}\right)^{2} d x d s,
\end{aligned}
$$

which together with $a(x, \tau) \geq C>0$ gives

$$
\begin{aligned}
& \int_{0}^{+\infty}\left(u_{1}-u_{2}\right)^{2} d x+\int_{0}^{+\infty}\left(\varrho_{1}-\varrho_{2}\right)^{2} d x \\
& \quad \leq C \int_{0}^{\tau} \int_{0}^{+\infty}\left(1+\left|\left(r^{2} u_{2}\right)_{x}\right|\right)^{2}\left(\varrho_{1}-\varrho_{2}\right)^{2} d x d s+C \int_{0}^{\tau} \int_{0}^{+\infty}\left(u_{1}-u_{2}\right)^{2} d x d s .
\end{aligned}
$$

From $\left(r^{2} u_{2}\right)_{x} \in L^{2}\left([0, T], H^{1}([0,+\infty))\right)$ and Sobolev's embedding theorem, we have $\left(r^{2} u_{2}\right)_{x} \in L^{2}\left([0, T], L^{\infty}([0,+\infty))\right)$, then using Gronwall's inequality, we can prove that

$$
\varrho_{1}(x, t)=\varrho_{2}(x, t), \quad u_{1}(x, t)=u_{2}(x, t) .
$$

The proof of the uniqueness is complete.

Competing interests

The authors declare that they have no competing interests.

\section{Authors' contributions}

$\mathrm{RL}$ proved and checked the theorem, and wrote the paper, JL rechecked the proofs. All authors read and approved the final manuscript. 


\section{Author details}

'College of Mathematics and Information Science, North China University of Water Resources and Electric Power, Zhengzhou, 450011 , P.R. China. ${ }^{2}$ Institute of Atmospheric Physics, Chinese Academy of Sciences, Beijing, 100029, P.R. China. ${ }^{3}$ College of Teacher Education, Quzhou University, Quzhou, 324000, P.R. China.

\section{Acknowledgements}

The authors thank the referee for the helpful comments and suggestions on the paper. The research of RX Lian is supported by NNSFC No. 11101145, China Postdoctoral Science Foundation No. 2012M520360, Doctoral Foundation of North China University of Water Sources and Electric Power No. 201032, Innovation Scientists and Technicians Troop Construction Projects of Henan Province. The research of J Liu is supported by NNSFC No. 11326140, the Doctoral Starting up Foundation of QuZhou University No. BSYJ201314.

\section{Received: 5 February 2015 Accepted: 17 January 2016 Published online: 22 February 2016}

\section{References}

1. Vaigant, VA, Kazhikhov, AV: On existence of global solutions to the two-dimensional Navier-Stokes equations for a compressible viscous fluid. Sib. Math. J. 36, 1108-1141 (1995)

2. Ducomet, B, Nečasová, Š: On the 2D compressible Navier-Stokes system with density-dependent viscosities. Nonlinearity 26, 1783-1797 (2013)

3. Gerbeau, JF, Perthame, B: Derivation of viscous Saint-Venant system for laminar shallow water; Numerical validation Discrete Contin. Dyn. Syst., Ser. B 1, 89-102 (2001)

4. Marche, F: Derivation of a new two-dimensional viscous shallow water model with varying topography, bottom friction and capillary effects. Eur. J. Mech. B, Fluids 26, 49-63 (2007)

5. Bresch, D, Desjardins, B: Existence of global weak solutions for a $2 \mathrm{D}$ viscous shallow water equations and convergence to the quasi-geostrophic model. Commun. Math. Phys. 238, 211-223 (2003)

6. Bresch, D, Desjardins, B: On the construction of approximate solutions for the $2 \mathrm{D}$ viscous shallow water model and for compressible Navier-Stokes models. J. Math. Pures Appl. 86, 362-368 (2006)

7. Fang, D, Zhang, T: Global solutions of the Navier-Stokes equations for compressible flow with density-dependent viscosity and discontinuous initial data. J. Differ. Equ. 222, 63-94 (2006)

8. Guo, Z, Li, H, Xin, Z: Lagrange structure and dynamics for solutions to the spherically symmetric compressible Navier-Stokes equations. Commun. Math. Phys. 309, 371-412 (2012)

9. Jiang, S, Xin, Z, Zhang, P: Global weak solutions to 1D compressible isentropy Navier-Stokes with density-dependent viscosity. Methods Appl. Anal. 12, 239-252 (2005)

10. Lian, R, Guo, Z, Li, H: Dynamical behaviors of vacuum states for $1 \mathrm{D}$ compressible Navier-Stokes equations. J. Differ Equ. 248, 1926-1954 (2010)

11. Liu, J: Local existence of solution to free boundary value problem for compressible Navier-Stokes equations. Acta Math. Sci. 1(32), 1298-1320 (2012)

12. Liu, T, Xin, Z, Yang, T: Vacuum states for compressible flow. Discrete Contin. Dyn. Syst. 4, 1-32 (1998)

13. Okada, M, Nečasová, $\breve{S}$, Makino, T: Free boundary problem for the equation of one-dimensional motion of compressible gas with density-dependent viscosity. Ann. Univ. Ferrara Sez. VII (N.S.) 48, 1-20 (2002)

14. Vong, S, Yang, T, Zhu, C: Compressible Navier-Stokes equations with degenerate viscosity coefficient and vacuum. II. J. Differ. Equ. 192, 475-501 (2003)

15. Yang, T, Yao, Z, Zhu, C: Compressible Navier-Stokes equations with density-dependent viscosity and vacuum. Commun. Partial Differ. Equ. 26, 965-981 (2001)

16. Yang, T, Zhao, H: A vacuum problem for the one-dimensional compressible Navier-Stokes equations with density-dependent viscosity. J. Differ. Equ. 184, 163-184 (2002)

17. Yang, T, Zhu, C: Compressible Navier-Stokes equations with degenerate viscosity coefficient and vacuum. Commun. Math. Phys. 230, 329-363 (2002)

18. Mellet, A, Vasseur, A: On the barotropic compressible Navier-Stokes equations. Commun. Partial Differ. Equ. 32 431-452 (2007)

19. Mellet, A, Vasseur, A: Existence and uniqueness of global strong solutions for one-dimensional compressible Navier-Stokes equations. SIAM J. Math. Anal. 39, 1344-1365 (2008)

20. Ducomet, B, Nečasová, Š, Vasseur, A: On global motions of a compressible barotropic and selfgravitating gas with density-dependent viscosities. Z. Angew. Math. Phys. 61, 479-491 (2010)

21. Ducomet, B, Nečasová, Š, Vasseur, A: On spherically symmetric motions of a viscous compressible barotropic and selfgravitating gas. J. Math. Fluid Mech. 13, 191-211 (2011)

22. Guo, Z, Jiu, Q, Xin, Z: Spherically symmetric isentropic compressible flows with density-dependent viscosity coefficients. SIAM J. Math. Anal. 39, 1402-1427 (2008)

23. Jiu, Q, Wang, Y, Xin, Z: Stability of rarefaction waves to the 1D compressible Navier-Stokes equations with density-dependent viscosity. Commun. Partial Differ. Equ. 36, 602-634 (2011)

24. Jiu, Q, Xin, Z: The Cauchy problem for 1D compressible flows with density-dependent viscosity coefficients. Kinet. Relat. Models 1, 313-330 (2008)

25. Li, H, Li, J, Xin, Z: Vanishing of vacuum states and blow-up phenomena of the compressible Navier-Stokes equations. Commun. Math. Phys. 281, 401-444 (2008)

26. Lian, R, Liu, J, Li, H, Xiao, L: Cauchy problem for the one-dimensional compressible Navier-Stokes equations. Acta Math. Sci. 1(32), 315-324 (2012)

27. Xin, Z: Blowup of smooth solutions to the compressible Navier-Stokes equation with compact density. Commun. Pure Appl. Math. 51, 229-240 (1998) 